\title{
PROPERTIES OF QUASI-ASSOUAD DIMENSION
}

\author{
Ignacio García and Kathryn Hare \\ Universidad Nacional de Mar del Plata, Facultad de Ciencias Exactas y Naturales \\ Instituto de Investigaciones Físicas de Mar del Plata (CONICET) \\ Centro Marplatense de Investigaciones Matemáticas (CIC) \\ Mar del Plata 7600, Argentina; nacholma@gmail.com \\ University of Waterloo, Department of Pure Mathematics \\ Waterloo, Ont., Canada N2L 3G1; kehare@uwaterloo.ca
}

\begin{abstract}
The connections between quasi-Assouad dimension and tangents are studied. We apply these results to the calculation of the quasi-Assouad dimension for a class of planar selfaffine sets. We also show that sets with decreasing gaps have quasi-Assouad dimension 0 or 1 and exhibit an example of a set in the plane whose quasi-Assouad dimension is smaller than that of its projection onto the $x$-axis, showing that quasi-Assouad dimension may increase under Lipschitz mappings. Moreover, for closed sets, we show that the Hausdorff dimension is an upper bound for the quasi-lower Assouad dimension.
\end{abstract}

\section{Introduction}

Recently, a number of authors have investigated the Assouad and lower-Assouad dimensions of subsets of $\mathbf{R}^{d}$; see $[6]$ and the many papers referenced there. These dimensions differ from the well-known Hausdorff and box dimensions as they provide information about the extreme behaviour of the local geometry of the set. The quasiAssouad dimensions give less extreme, but still local, geometric information. As in the case of Assouad dimensions, quasi-Assouad dimensions take into account relative scales locally at any position in the set, but the difference is that the 'minimal depth' of the relative scales increases as the size of the considered neighborhood decreases, and this has a moderating effect. These dimensions were introduced by Lü and Xi in [21] and shown to be an invariant quantity under quasi-Lipschitz maps, unlike the Assouad dimension. In this note, we develop further properties of the quasi-Assouad dimensions.

To give their definitions we first introduce notation. For a subset $A \subseteq \mathbf{R}^{d}$, let $N_{r}(A)$ be the least number of balls of radius $r$ needed to cover $A$ and let $N_{r, R}(E)=$ $\max _{x \in E} N_{r}(E \cap B(x, R))$. The upper and lower box dimensions, denoted $\overline{\operatorname{dim}}_{B} E$ and $\underline{\operatorname{dim}}_{B} E$ respectively, are given by

$$
\overline{\operatorname{dim}}_{B} E=\limsup _{r \rightarrow 0} \frac{\log N_{r}(E)}{\log r}, \underline{\operatorname{dim}}_{B} E=\liminf _{r \rightarrow 0} \frac{\log N_{r}(E)}{\log r}
$$

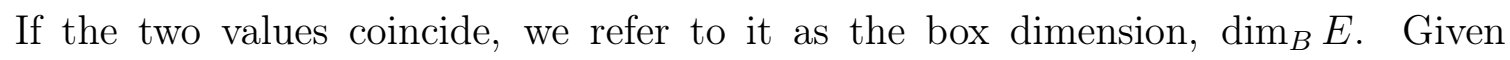
$0 \leq \delta<1$, define

$$
\begin{aligned}
& \overline{h_{E}}(\delta) \\
& =\inf \left\{\alpha \geq 0: \exists b, c>0 \text { such that } \forall 0<r \leq R^{1+\delta} \leq R \leq b, N_{r, R}(E) \leq c\left(\frac{R}{r}\right)^{\alpha}\right\}
\end{aligned}
$$

https://doi.org/10.5186/aasfm.2021.4618

2010 Mathematics Subject Classification: Primary 28A80, 28A78.

Key words: Assouad dimension, weak tangents, orthogonal projections. 
and

$\underline{h_{E}}(\delta)$

$$
=\sup \left\{\alpha \geq 0: \exists b, c>0 \text { such that } \forall 0<r \leq R^{1+\delta} \leq R \leq b, N_{r, R}(E) \geq c\left(\frac{R}{r}\right)^{\alpha}\right\} \text {. }
$$

These functions are monotone and by taking limits we obtain the quasi-Assouad dimension of $E, \operatorname{dim}_{q A} E$, and the quasi-lower Assouad dimension of $E, \operatorname{dim}_{q L} E$ :

$$
\operatorname{dim}_{q A} E=\lim _{\delta \rightarrow 0} \overline{h_{E}}(\delta), \quad \operatorname{dim}_{q L} E=\lim _{\delta \rightarrow 0} \underline{h_{E}}(\delta) .
$$

The Assouad and the lower-Assouad dimensions are given by

$$
\operatorname{dim}_{A} E=\overline{h_{E}}(0), \quad \operatorname{dim}_{L} E=\underline{h_{E}}(0) .
$$

For any bounded set $E$ these dimensions are ordered in the following way:

$$
\operatorname{dim}_{L} E \leq \operatorname{dim}_{q L} E \leq \underline{\operatorname{dim}}_{B} E \leq \overline{\operatorname{dim}}_{B} E \leq \operatorname{dim}_{q A} E \leq \operatorname{dim}_{A} E .
$$

It is known that all these dimensions coincide for self-similar sets satisfying the open set condition, but for more general sets, strict inequalities are possible throughout, cf. $[6,21]$ and Example 11 in Section 3. Moreover, in that section we show that inequality $\operatorname{dim}_{q L} E \leq \operatorname{dim}_{H} E$ holds for closed sets. This inequality is not true in general, since $\operatorname{dim}_{H} \mathbf{Q}=0$ but $\operatorname{dim}_{q L} \mathbf{Q}=1$.

Generalizations of tangents of a set are a useful concept when considering the Assouad dimension. These are essentially limits, in the Hausdorff metric, of sequences of magnifications of local parts of the set. Tangents often have simpler structure than the original set and their Assouad dimensions are always lower bounds for the Assouad dimension of the original set $[6,22,23]$. In Section 2, we show that if the convergence to the tangent is sufficiently fast, and the lower-Assouad and Assouad dimensions of the tangent coincide, then this value is a lower (upper) bound for the quasi-(lower) Assouad dimension of the original set. From this, we show that the quasi-(lower) Assouad dimensions of a class of self-affine carpets are the same as their Assouad dimensions.

In Section 3 we observe that if $\overline{\operatorname{dim}}_{B} E=0$, then the same is true for the quasiAssouad (but not necessarily, the Assouad) dimension of $E$ and show that the quasiAssouad dimension of a sequence in $\mathbf{R}$ with decreasing gaps is either 0 or 1 . This same dichotomy was shown to hold for Assouad dimensions (although not necessarily with the same value for the Assouad and quasi-Assaoud dimensions) in [17]. We also give an example to illustrate that, like the Assouad dimension (but not the Hausdorff or box dimensions), the quasi-Assouad dimension can rise when taking projections.

\section{Quasi-Assouad dimension and tangent structure}

In this section we find bounds on the quasi-Assouad dimension of a set by dimensions of its 'tangents'. We begin by recalling some definitions and results. Given $X, Y \subset \mathbf{R}^{d}$ compact subsets, their Hausdorff distance is

$$
\operatorname{dist}_{H}(X, Y)=\max \left\{p_{H}(X, Y), p_{H}(Y, X)\right\},
$$

where

$$
p_{H}(X, Y)=\sup _{x \in X} \inf _{y \in Y}\|x-y\| .
$$


Definition 1. Let $F$ and $\hat{F}$ be compact subsets of $\mathbf{R}^{d}$. We say that $\hat{F}$ is a weak tangent of $F$ if there is a compact subset $X \subset \mathbf{R}^{d}$, that contains both $F$ and $\hat{F}$, and a sequence of bi-Lipschitz maps $T_{k}: \mathbf{R}^{d} \rightarrow \mathbf{R}^{d}$, with Lipschitz constants $a_{k}, b_{k}$ satisfying

$$
a_{k}\|x-y\| \leq\left\|T_{k}(x)-T_{k}(y)\right\| \leq b_{k}\|x-y\|
$$

and $\sup b_{k} / a_{k}<\infty$, such that $\operatorname{dist}_{H}\left(T_{k}(F) \cap X, \hat{F}\right) \rightarrow 0$ as $k \rightarrow \infty$. In the case that the contraction ratios, $b_{k}$, are unbounded we call $\hat{F}$ a generalized tangent of $F$.

The usefulness of tangents is that they can be used to obtain lower bounds for the Assouad dimension of the original set, namely

Theorem 1. If $\hat{F}$ is a weak tangent of $F$, then

$$
\operatorname{dim}_{A} \hat{F} \leq \operatorname{dim}_{A} F
$$

If, in addition, there is some $\theta>0$ such that for all $r>0$ and $x \in \hat{F}$ there is some $y \in \hat{F}$ such that $B(y, r \theta) \subseteq B(x, r) \cap X$, then

$$
\operatorname{dim}_{L} F \leq \operatorname{dim}_{L} \hat{F}
$$

The above result is due to Fraser [6, Prop. 7.7]. A version using similarities to define tangents (the usual definition) was previously obtained by Mackay and Tyson, see [22, Prop. 2.9] or [23, Prop. 6.1.5].

There is no loss of generality in assuming that $X=[0,1]^{d}$ since the Assouad dimension of any set can be characterized by its tangents in this sense. To be specific (4) $\operatorname{dim}_{A} F=\max \left\{\operatorname{dim}_{H} \hat{F}: \hat{F}\right.$ is a generalized tangent of $F$ with $\left.X=[0,1]^{d}\right\}$.

We refer the reader to [3], [15], [16] and [19], noting that the terminology there is quite different: microsets and star dimension are used instead of tangents and Assouad dimension. Moreover, for the lower-Assouad dimension, an analogous result holds:

$$
\operatorname{dim}_{L} F=\min \left\{\operatorname{dim}_{H} E: E \in \mathcal{G}_{F}\right\}
$$

where the elements in $\mathcal{G}_{F}$ are weak tangents intersecting $(0,1)^{d}$ and obtained by magnifications with homotheties; see [9].

We call the set $\hat{F}$ a pseudo-tangent of $F$ if there is a sequence of bi-Lipschitz maps $T_{k}$, as above, with $\sup b_{k}=\infty$, such that we have the 'one-sided' pseudo-distance, $p_{H}\left(\hat{F}, T_{k}(F)\right) \rightarrow 0$ as $k \rightarrow \infty$. The convenience of this definition is that it does not require the intersection with the set $X$ or the two-sided comparison of distance, and still the inequality $\operatorname{dim}_{A} \hat{F} \leq \operatorname{dim}_{A} F$ holds in this case; see [8], where the definition and proof are made for similarities, but the same proof applies in the bi-Lipschitz setting.

Returning to the quasi-Assouad dimension, we first give an example where (2) fails when Assouad is replaced by quasi-Assouad dimension. The following result is useful for this task; a more general version in $\mathbf{R}^{d}$ was obtained recently [14], but here we include a different proof.

Proposition 2. A subset $F \subseteq \mathbf{R}$ has Assouad dimension 1 if and only if $[0,1]$ is a generalized tangent of $F$.

Proof. By (2), the Assouad dimension of any tangent is a lower bound for the Assouad dimension of the set. On the other hand, by [23, Thm. 5.1.8], the assumption that $\operatorname{dim}_{A} F=1$ is equivalent to the fact that $F$ is not uniformly disconnected. Thus for each $k$ there are distinct points, $z_{0}^{k}, \ldots, z_{n}^{k} \in F$, such that $\left|z_{i}-z_{i+1}\right|<\left|z_{0}-z_{n}\right| / k$, 
where $z_{0}<z_{i}<z_{n}$ and $\left|z_{0}-z_{n}\right|$ goes to 0 . The last condition ensures that $[0,1]$ is a generalized tangent (and not just a weak tangent). we have

Let $T_{k}$ be the affine transformation that maps $\left[z_{0}, z_{n}\right]$ to $[0,1]$. For $x_{i}=T_{k}\left(z_{i}\right)$

$$
\left|x_{i}-x_{i+1}\right|=\frac{\left|z_{i}^{k}-z_{i+1}^{k}\right|}{\left|z_{n}^{k}-z_{0}^{k}\right|}<\frac{1}{k}
$$

and hence for $0 \leq j<k$ each interval $[j / k,(j+1) / k)$ contains at least one of the $x_{i}$ 's. This shows the sets $T_{k}\left(\left\{z_{0}^{k}, \ldots, z_{n}^{k}\right\}\right)$ converge in the Hausdorff metric to $[0,1]$ and therefore, $\operatorname{dist}_{H}\left(T_{k}(F) \cap[0,1],[0,1]\right) \rightarrow 0$ as $k \rightarrow \infty$.

Corollary 3. If $F \subseteq \mathbf{R}$ has $\operatorname{dim}_{q A} F<1=\operatorname{dim}_{A} F$, then $\hat{F}=[0,1]$ is a generalized tangent of $F$ with

$$
1=\operatorname{dim}_{H} \hat{F}=\operatorname{dim}_{q A} \hat{F}>\operatorname{dim}_{q A} F .
$$

More generally, from (2) and (4), any compact set $F$ has a generalized tangent $\hat{F}$ for which $\operatorname{dim}_{H} \hat{F}=\operatorname{dim}_{A} \hat{F}=\operatorname{dim}_{A} F$. This shows that inequality (2) fails when Assouad is replaced by quasi-Assouad dimension if we consider a set $F$ with $\operatorname{dim}_{q A} F<\operatorname{dim}_{A} F$. Also, for the lower-Assouad dimension case, by (5) there is a weak tangent $E$ of $F$ such that $\operatorname{dim}_{L} F=\operatorname{dim}_{H} E \geq \operatorname{dim}_{q L} E$, where the last inequality holds by Proposition 10 in Section 3.2. So, if $\operatorname{dim}_{L} F<\operatorname{dim}_{q L} F$, then inequality (3) may also fail when lower-Assouad is replaced by quasi-lower Assouad. Below, in Theorem 6, we show that inequalities (2) and (3) hold for quasi and quasi-lower Assouad dimensions provided we restrict the family of tangents; the analog of (3) requires a similar hypothesis. However, in general it is not possible to characterize the quasi or quasi-lower Assouad dimension of a set in terms of the (Hausdorff) dimension of its tangents. The following example is useful to illustrate this fact.

Example 4. Fix $0<\alpha<1$ and for each $k \geq 1$ put $D^{k}=\left\{\left(i_{1}, \ldots, i_{k}\right): 1 \leq i_{j} \leq\right.$ $\left.2^{j}, 1 \leq j \leq k\right\}$. We construct the set inductively, beginning with $I_{\phi}=[0,1]$ at step 0 . Having constructed the step $k-1$ Cantor intervals, $I_{i_{1} \ldots i_{k-1}}$, for $\left(i_{1}, \ldots, i_{k-1}\right) \in D^{k-1}$, to construct the Cantor intervals of step $k$ we take the $2^{k}$ closed subintervals, $I_{i_{1} \ldots i_{k-1} l}$, $1 \leq l \leq 2^{k}$, uniformly distributed inside $I_{i_{1} \ldots i_{k-1}}$, with equal lengths $2^{-k / \alpha}\left|I_{i_{1} \ldots i_{k-1}}\right|$. The Cantor-like set $F_{\alpha}$ is given by

$$
F_{\alpha}=\bigcap_{k \geq 1} \bigcup_{\mathbf{i} \in D^{k}} I_{\mathbf{i}}
$$

Example 1.17 from [21] is a special case and it follows from the arguments given there that $\operatorname{dim}_{q A} F_{\alpha}=\operatorname{dim}_{q L} F_{\alpha}=\alpha$, while $\operatorname{dim}_{A} F_{\alpha}=1$. Moreover, since the ratio between the lengths of a complementary open interval from step $k$ and a closed interval from the same step goes to infinity, it follows that $\operatorname{dim}_{L} F_{\alpha}=0$.

Moreover, this example turns out to be quite pathological with respect to tangents.

Proposition 5. Any generalized tangent of the set $F_{\alpha}$ of the example above is either a finite set or an interval.

Proof. For notational ease, we will omit the subscript $\alpha$. Let $\hat{F}$ be a generalized tangent of $F$ and denote by $T_{k}$ the associated bi-Lipschitz maps. By a gap of $\hat{F}$ we mean a bounded complementary (maximal) open interval of the complement of $\hat{F}$. If $\hat{F}$ has no gaps, then it is an interval (which may be a singleton). So, assume that 
$\hat{F}$ contains at least one gap. Choose one of maximal length, say $J=(a, b)$, where $a, b \in \hat{F}$. Below we show that $i$ ) if $x \in \hat{F}$ and $x<a$, then $a-x \geq|J|$, or $i i)$ if $x \in \hat{F}$ and $x>b$, then $x-b \geq|J|$. This statements imply, by the maximality of $J$, that $\hat{F}$ is a finite equidistributed set in some subinterval of $[0,1]$.

We show only $i$ ) since $i i$ ) follows by a symmetric argument. So assume that $x<a$ with $x \in \hat{F}$. One consequence of the Hausdorff convergence is that for each sufficiently small $\epsilon>0$ (much smaller than $|J|$ and $a-x$ ), and all sufficiently large $k$, the sets $(a-\epsilon, a+\epsilon)$ and $(b-\epsilon, b+\epsilon)$ contain points of $T_{k}(F)$, while $(a+\epsilon, b-\epsilon) \cap T_{k}(F)=\emptyset$. Hence there is a gap $G_{k}$ of $F$ from some step $k$ in the construction of $F$ such that

$$
(a+\epsilon, b-\epsilon) \subseteq T_{k}\left(G_{k}\right) \subseteq(a-\epsilon, b+\epsilon) .
$$

Suppose $I_{k}^{L}$ is the closed interval from step $k$ in the construction of $F$ that shares an endpoint with $G_{k}$ and is placed on its left. The set $F$ has the property that $\left|I_{k}^{L}\right| /\left|G_{k}\right| \rightarrow 0$ and as the maps $T_{k}$ are bi-Lipschitz with constants satisfying $\sup b_{k} / a_{k}$ $<\infty$, this ensures that $T_{k}\left(I_{k}^{L}\right) \subseteq(a-2 \epsilon, a+2 \epsilon)$ for large enough $k$. Note that the gap $G_{k}^{L}$, adjacent to $I_{k}^{L}$ but on its left, and whose existence is guaranteed because of the existence of $x$ and the choice of $\epsilon$, is a gap from some step $\tilde{k}<k$. Note also that the lengths $g_{k-1}$ and $g_{k}$ of any gaps from steps $k-1$ and $k$ verify $g_{k} / g_{k-1} \rightarrow 0$ as $k \rightarrow \infty$. In particular, for every $k$ sufficiently large we get $b_{k}\left|G_{k}\right| \leq a_{k}\left|G_{k}^{L}\right|$.

Thus

$$
|J|-2 \epsilon \leq\left|T_{k}\left(G_{k}\right)\right| \leq\left|T_{k}\left(G_{k}^{L}\right)\right|
$$

so any point in $\hat{F}$ to the left of $a$, and not contained in $(a-2 \epsilon, a+2 \epsilon)$, must be at least distance $|J|-2 \epsilon$ from $a$ for some constant $c>0$. But since $\epsilon$ can be made arbitrarily small, an easy argument shows that $\hat{F} \cap(a-2 \epsilon, a+2 \epsilon)=\{a\}$, and therefore we conclude that $i$ ) holds by letting $\epsilon \rightarrow 0$.

Note that the above example also illustrates that there is no way to select a subfamily from the tangents to $F$ so that the identity (4) remains valid for the quasiAssouad dimension. However, we can extend Theorem 1 above to the quasi-Assouad dimensions if we restrict to tangents for which the convergence is sufficiently quick.

Definition 2. We say that the generalized tangent $\hat{F}$ to the set $F$ is a generalized fast tangent if the following decay condition is satisfied: there are constants $C, \epsilon>0$ such that

$$
\operatorname{dist}_{H}\left(T_{k}(F) \cap[0,1]^{d}, \hat{F}\right) \leq C b_{k}^{-\epsilon},
$$

where $T_{k}$ and $b_{k}$ are as in the definition of a generalized tangent. In this case we say that $\hat{F}$ is a generalized fast tangent of order $\epsilon$. We similarly define fast pseudotangents by the requirement that the pseudo-tangent $\hat{F}$ verifies $p_{H}\left(\hat{F}, T_{k}(F)\right) \leq C b_{k}^{-\epsilon}$.

The relation between tangents and quasi-Assouad dimensions is given in the next result, where we have chosen to weaken some hypotheses for clarity of the exposition; see Remark 2 for more general statements.

Theorem 6. Suppose $\hat{F} \subset \mathbf{R}^{d}$ is a non-empty, generalized fast tangent of $F \subset$ $\mathbf{R}^{d}$ given by bi-Lipschitz maps $T_{k}$ with Lipschitz constants $a_{k}, b_{k}$ satisfying $\sup b_{k}=$ $\infty$ and $\sup b_{k} / a_{k}<\infty$.

(i) For the quasi-Assouad dimension of $F$ we have the lower bound

$$
\underline{\operatorname{dim}}_{B} \hat{F} \leq \operatorname{dim}_{q A} F \text {. }
$$


If, in addition, there is some $C^{\prime}$ such that $b_{k+1} \leq C^{\prime} b_{k}$, then

$$
\operatorname{dim}_{q A} \hat{F} \leq \operatorname{dim}_{q A} F .
$$

(ii) Assume that $\hat{F}$ contains an interior point of $[0,1]^{d}$. Then, for the quasi-lower Assouad dimension we have the upper bound

$$
\operatorname{dim}_{q L} F \leq \overline{\operatorname{dim}}_{B} \hat{F} .
$$

If, in addition, there is some $C^{\prime}$ such that $b_{k+1} \leq C^{\prime} b_{k}$ and furthermore, there is $\theta>0$ such that for any $r \in(0,1]$ and $x \in \hat{F}$ there is $y \in \hat{F}$ such that $B(y, r \theta) \subset B(x, r) \cap[0,1]^{d}$, then

$$
\operatorname{dim}_{q L} F \leq \operatorname{dim}_{q L} \hat{F} .
$$

Remark 1. In order to better understand how the inhomogeneity of a set depends on the scale, Fraser and $\mathrm{Yu}[12]$ considered the refined parametric variants of the quasi-Assouad and quasi-lower Assouad dimensions, $\operatorname{dim}_{A}^{\theta} F$ and $\operatorname{dim}_{L}^{\theta} F$, for $\theta \in(0,1)$, known as the Assouad spectrum and lower spectrum of $E$ respectively; see [12] for the precise definitions. Similar statements as in Theorem 6 can be made for $\operatorname{dim}_{A}^{\theta} F$ and $\operatorname{dim}_{L}^{\theta} F$, where the allowable $\theta$ depend on the choice of $\varepsilon$. For example, if $\hat{F}$ is a generalized fast tangent of order $\epsilon$ such that $\underline{\operatorname{dim}}_{B} \hat{F}=s$, then $\operatorname{dim}_{A}^{\theta} F \geq s$ for any $1 /(1+\epsilon) \leq \theta<1$. We leave the technical details for the reader.

Notation: When we write $x_{k} \approx X_{k}$ we mean there are positive constants $a, b$ such that $a X_{k} \leq x_{k} \leq b X_{k}$ for all $k$.

Proof. (i) Let $\underline{\operatorname{dim}}_{B} \hat{F}=s$. We may assume $s>0$, else the result is trivial. Temporarily fix $\eta>0$. Then

$$
N_{r}(\hat{F}) \geq r^{-(s-\eta)}
$$

for all sufficiently small $r$.

Since $\hat{F}$ is a generalized fast tangent, there are constants $C, \epsilon>0$ such that $\operatorname{dist}_{H}\left(T_{k}(F) \cap[0,1]^{d}, \hat{F}\right) \leq C b_{k}^{-\epsilon}$. Put $r_{k}=C b_{k}^{-\varepsilon}$, let $R=\operatorname{diam} \hat{F}$ and pick any $y_{0} \in \hat{F}$. Then, for each $k$ we can find $m=m_{k}\left(\approx r_{k}^{-(s-\eta)}\right)$ points, $y_{1}, \ldots, y_{m} \in$ $\hat{F} \cap B\left(y_{0}, R\right)$ that are $3 r_{k}$-separated. The assumption on $\hat{F}$ implies, in particular, that $p_{H}\left(\hat{F}, T_{k}(F)\right) \leq r_{k}$, and this ensures that we can choose $x_{0}, x_{1}, \ldots, x_{m} \in T_{k}(F)$ such that $\left\|x_{i}-y_{i}\right\| \leq r_{k}$ for each $i=0, \ldots, m$. We have $x_{i} \in B\left(x_{0}, R+2 r_{k}\right)$ and $\left\|x_{i}-x_{j}\right\| \geq r_{k}$ for all $1 \leq i \neq j \leq m$.

For $0 \leq i \leq m$, we consider the preimages $z_{i}=T_{k}^{-1}\left(x_{i}\right) \in F$, which satisfy

$$
z_{i} \in B\left(z_{0}, \frac{1}{a_{k}}\left(R+2 r_{k}\right)\right) \text { and }\left\|z_{i}-z_{j}\right\| \geq \frac{r_{k}}{b_{k}} .
$$

This shows that

$$
N_{\frac{r_{k}}{b_{k}}}\left(F \cap B\left(z_{0}, \frac{1}{a_{k}}\left(R+2 r_{k}\right)\right)\right) \geq m .
$$

Note that $m \approx\left(\frac{\left(R+2 r_{k}\right) / a_{k}}{r_{k} / b_{k}}\right)^{s-\eta}$ (and hence $\operatorname{dim}_{A} F \geq s$ ). An easy calculation shows

$$
\frac{r_{k}}{b_{k}} \leq\left(\frac{1}{a_{k}}\left(R+2 r_{k}\right)\right)^{1+\varepsilon / 2}
$$

for large $k$, and, of course, $\left(R+2 r_{k}\right) / a_{k} \rightarrow 0$, consequently $\operatorname{dim}_{q A} F \geq s-\eta$. As $\eta$ $>0$ is arbitrary, $\operatorname{dim}_{q A} F \geq s$.

Now suppose that $\operatorname{dim}_{q A} \hat{F}=t$ and there is some $C^{\prime}$ such that $b_{k+1} \leq C^{\prime} b_{k}$ for all $k$. Again, temporarily fix $\eta>0$. Then there is some $0<\delta<1$ and arbitrarily small 
$r, R$ with $r \leq R^{1+\delta}$ and $y_{0} \in \hat{F}$ such that $N_{r}\left(B\left(y_{0}, R\right) \cap \hat{F}\right) \geq(R / r)^{t-\eta}$. Choose $k$ such that $b_{k+1}^{-\varepsilon} \leq R \leq b_{k}^{-\varepsilon}$. As above, we deduce that for suitable $z_{0} \in F$ we have

$$
N_{\frac{r}{b_{k}}}\left(F \cap B\left(z_{0}, \frac{1}{a_{k}}(R+2 r)\right)\right) \geq\left(\frac{R}{r}\right)^{t-\eta} .
$$

Since $R \approx b_{k}^{-\varepsilon}$ and $r \leq R^{1+\delta}$, one can easily verify that $r / b_{k} \geq\left((R+2 r) / a_{k}\right)^{1+\sigma}$ for a choice of $\sigma>0$ (depending on $\delta$ and $\varepsilon$ ). It follows that $\operatorname{dim}_{q A} F \geq t$.

(ii) Let $\overline{\operatorname{dim}}_{B} \hat{F}=s$. Given any $\eta>0$ we have $N_{r_{k}}(\hat{F}) \leq r_{k}^{-(s+\eta)}$ if $k$ is sufficiently large, where as before $r_{k}=C b_{k}^{-\epsilon}$ with $C$ and $\epsilon$ given by the definition of the generalized fast tangent. The generalized fast tangent hypothesis implies that for each $x \in T_{k}(F) \cap[0,1]$ there is some $\hat{x} \in \hat{F}$ such that $\|x-\hat{x}\| \leq r_{k}$ and this ensures that $N_{3 r_{k}}\left(T_{k}(F) \cap[0,1]^{d}\right) \leq r_{k}^{-(s+\eta)}$.

Also, by hypothesis, there are $\hat{y} \in \hat{F}$ and $\theta>0$ such that $B(\hat{y}, 2 \theta) \subset[0,1]^{d}$ and thus, for $k$ sufficiently large there is a point $y_{k} \in T_{k}(F)$ so that $B\left(y_{k}, \theta\right) \subset[0,1]^{d}$. It follows that

$$
N_{3 r_{k}}\left(T_{k}(F) \cap B\left(y_{k}, \theta\right)\right) \leq r_{k}^{-(s+\eta)} .
$$

Defining $r=3 r_{k} a_{k}^{-1}, R=\theta b_{k}^{-1}$ and $z_{k}=T_{k}^{-1}\left(y_{k}\right) \in F$, we get

$$
N_{r}\left(F \cap B\left(z_{k}, R\right)\right) \leq\left(\frac{R}{r}\right)^{s+\eta}
$$

(and hence $\operatorname{dim}_{L} F \leq s$ ). It is easily seen that $r \leq R^{1+\epsilon / 2}$, therefore, $\operatorname{dim}_{q L} F \leq s$.

Finally, suppose that $\operatorname{dim}_{q L} \hat{F}=t$, so given $\eta>0$ there is some $0<\delta<1$ and arbitrarily small $r, R$ with $r \leq R^{1+\delta}$ and $\hat{y} \in \hat{F}$ such that

$$
N_{r}(B(\hat{y}, R) \cap \hat{F}) \leq(R / r)^{t+\eta} \text {. }
$$

The geometric condition involving $\theta$ allows us to assume that $B(\hat{y}, R) \subset[0,1]^{d}$. Now choose $k$ such that $C b_{k+1}^{-\epsilon} \leq r \leq C b_{k}^{-\epsilon}$, where $C, \epsilon$ are as before. Then, there is $y \in T_{k}(F) \cap[0,1]^{d}$ such that $\|y-\hat{y}\| \leq C b_{k}^{-\epsilon}$ and moreover, $B\left(y, \frac{1}{2} R\right) \subset[0,1]^{d}$ (for $k$ sufficiently large). Defining $r_{k}=3 C b_{k}^{-\epsilon}$ and $R_{k}=R / 2$, it follows that for $k$ sufficiently large,

$$
N_{r_{k}}\left(T_{k}(F) \cap B\left(y, R_{k}\right)\right) \leq N_{r}(\hat{F} \cap B(\hat{y}, R)) \leq(R / r)^{t+\eta} \approx\left(\frac{R_{k}}{r_{k}}\right)^{t+\eta},
$$

where in the last equivalence we used the fact that $b_{k+1} \leq C^{\prime} b_{k}$ for some constant $C^{\prime}$. For an appropriate $z_{k} \in F$ and a constant $C^{\prime \prime}$, we get

$$
N_{\frac{r_{k}}{a_{k}}}\left(F \cap B\left(z, \frac{R_{k}}{b_{k}}\right)\right) \leq C^{\prime \prime}\left(\frac{R_{k} / b_{k}}{r_{k} / a_{k}}\right)^{t+\eta} .
$$

It is easily seen that $r_{k} / a_{k} \leq\left(R_{k} / b_{k}\right)^{1+\delta / 2}$ for $k$ sufficiently large, and therefore $\operatorname{dim}_{q L} F \leq \operatorname{dim}_{q L} \hat{F}$.

Corollary 7. Suppose that $\hat{F} \subset \mathbf{R}^{d}$ is a generalized fast tangent of $F$ such that $\operatorname{dim}_{B} \hat{F}=s$. Then $\operatorname{dim}_{q L} F \leq s \leq \operatorname{dim}_{q A} F$ whenever $\hat{F}$ contains an interior point of $[0,1]^{d}$.

Remark 2. The statements from Theorem 6 can be improved.

a) Part (i) only needs the one-sided hypothesis, $p_{H}\left(\hat{F}, T_{k}(F)\right) \leq C b_{k}^{-\epsilon} \rightarrow 0$, i.e., $\hat{F}$ is a fast pseudo-tangent of $F$. This is immediate from the proof. 
b) A quick inspection of the proof of (ii) shows that we have $\operatorname{dim}_{L} F \leq \overline{\operatorname{dim}}_{B} \hat{F}$ even if the convergence to the generalized tangent is not fast. Obviously by (5), this bound can be improved by replacing the upper box dimension by the Hausdorff dimension.

Remark 3. Consider the following simple example. Suppose that $F \subset[0,1]$ and that $(a, b) \subset[0,1]$ with $a, b \in F$, but $(a, b) \cap F=\emptyset$. By considering $T_{k} x=2^{k}(x-a)$, it is easily seen that $\hat{F}=\{0\}$ is a fast tangent of $F$, so, unless $\operatorname{dim}_{q L} F=0$, the conclusion in (ii) is false in this case. This example illustrates that for the quasilower Assouad dimension, an additional hypothesis that ensures the tangent 'carries' information about the interior of $F$ is necessary. In the statement of (ii), we have chosen to put this hypothesis directly on $\hat{F}$. Alternatively, we could have put an additional hypothesis on the approximations of the tangent, for example requiring that $p_{H}\left(F \cap B\left(z_{k}, C a_{k}^{-1}\right), \hat{F}\right) \leq C b_{k}^{-\epsilon}$, where $z_{k} \in F$. The proof is a slight modification of the one given here.

As an application of our results, we calculate the quasi-Assouad dimensions of a class of planar self-affine sets. Recall that an iterated function system (IFS) is a family $\left\{f_{1}, \ldots, f_{m}\right\}$ of contractions $f_{i}: \mathbf{R}^{d} \rightarrow \mathbf{R}^{d}$, and that the attractor of the IFS is the unique non-empty compact set $E$ that satisfies the identity

$$
E=\bigcup_{i=1}^{m} f_{i}(E) .
$$

If the maps of the IFS are contracting similarities (affine maps), the attractor is called a self-similar set (self-affine set, respectively).

In [6, Sec. 2.3], Fraser determines the (lower) Assouad dimensions of self-affine carpets that are the attractor of an IFS in the extended Lalley-Gatzouras and Barański classes. These families, denoted $E$, contain the Bedford-McMullen class and are generated by an IFS with maps of the form $S_{i}(x, y)=\left(c_{i} x, d_{i} y\right)+\left(a_{i}, b_{i}\right)$, for some $c_{i}, d_{i} \in(0,1), 1 \leq i \leq m$, where $c_{i} \neq d_{i}$ for at least one $i$. (See [6] for their complete definitions.) Let $\pi_{1}$ denote the projection onto the $x$-axis and $\pi_{2}$ the projection onto the $y$-axis. Let $\operatorname{Slice}_{1, i}(E)$ (resp., Slice $\left._{2, i}(E)\right)$ be the vertical (horizontal) slice of $E$ through the fixed point of $S_{i}$. Fraser proves that if the self-affine carpet $E$ is of mixed type, i.e., there are $i \neq i^{\prime}$ such that $c_{i}>d_{i}$ and $c_{i^{\prime}}<d_{i^{\prime}}$, then

$$
\begin{aligned}
& \operatorname{dim}_{A} E=\max _{i} \max _{k=1,2}\left(\operatorname{dim}_{B} \pi_{k}(E)+\operatorname{dim}_{B} \operatorname{Slice}_{k, i}(E)\right), \\
& \operatorname{dim}_{L} E=\min _{i} \min _{k=1,2}\left(\operatorname{dim}_{B} \pi_{k}(E)+\operatorname{dim}_{B} \operatorname{Slice}_{k, i}(E)\right) .
\end{aligned}
$$

Applying Corollary 7 we obtain the following result. We observe that the quasiAssouad dimension of the Bedford-McMullen carpets was obtained in [21].

Proposition 8. For the above carpets we have $\operatorname{dim}_{q A} E=\operatorname{dim}_{A} E$, and similarly for the quasi-lower Assouad dimension.

Proof. To see this, we give the following sketch of the proof (see [6, Sec 7.2] for more details on the definitions). We assume

$$
\max _{i} \max _{k=1,2}\left(\operatorname{dim}_{B} \pi_{k}(E)+\operatorname{dim}_{B} \operatorname{Slice}_{k, i}(E)\right)=\operatorname{dim}_{B} \pi_{1}(E)+\operatorname{dim}_{B} \operatorname{Slice}_{1, i}(E)
$$

for some $1 \leq i \leq m$, that now we fix. Consider the approximate square $Q_{k}(i, j)$, centred at the point $\bigcap_{l \geq 1} S_{j}^{k} \circ S_{i}^{l}\left([0,1]^{2}\right)$, with height $d_{j}^{k}$ and width $c_{j}^{k} c_{i}^{l(k)}$, where $l(k)$ 
is an integer chosen so that

$$
c_{j}^{k} c_{i}^{l(k)+1} \leq d_{j}^{k} \leq c_{j}^{k} c_{i}^{l(k)} .
$$

Take the maps $T_{k}$ that stretch by $d_{j}^{-k}$ in height and by $\left(c_{j}^{k} c_{i}^{l(k)}\right)^{-1}$ in width, and map the corner of $Q_{k}$ to the origin. One can check these maps satisfy the required Lipschitz properties with $b_{k}=d_{j}^{-k}$ and $a_{k}=\left(c_{j}^{k} c_{i}^{l(k)}\right)^{-1}$. Take $F_{i}=\pi_{1}(E) \times \pi_{2}\left(\operatorname{Slice}_{1, i}(E)\right)$. This is a product of two self-similar sets satisfying the open set condition and hence

$$
\operatorname{dim}_{B} F_{i}=\operatorname{dim}_{B} \pi_{1}(E)+\operatorname{dim}_{B} \operatorname{Slice}_{1, i}(E) .
$$

From the structure of the carpet, and since $l(k)>k \log _{c_{i}}\left(d_{j} / c_{j}\right)$, it can be seen that

$$
\operatorname{dist}_{H}\left(T_{k}\left(Q_{k}\right), F_{i}\right) \leq \max _{n} d_{n}^{l(k)} \leq d_{j}^{k \beta}
$$

for a suitable $\beta>0$. Appealing to the corollary gives the result.

We finish the section with the following remark on the quasi-Assouad dimension of self-similar sets.

Remark 4. For self-similar sets, the weak separation property (WSP) is a separation property (on an IFS) that, although weaker than the classical open set condition, ensures nice properties on the attractor; see [24] for the definition. This property has been essential to describe the behaviour of the Assouad dimension of self-similar sets. If it holds, then $\operatorname{dim}_{B} E=\operatorname{dim}_{A} E$, so in particular, $\operatorname{dim}_{q A} E=\operatorname{dim}_{B} E$. Moreover, if it does not hold, then $\operatorname{dim}_{A} E \geq 1$; see [8]. These results establish, in $\mathbf{R}$, the precise dichotomy that $\operatorname{dim}_{A} E$ is either $\operatorname{dim}_{B} E$ or 1 depending on whether the WSP holds or not.

In absence of the WSP, the behaviour of the quasi-Assouad dimension is unknown. However, it still remains valid in $\mathbf{R}$ that $\operatorname{dim}_{q A} E=\operatorname{dim}_{B} E$ in the case that the IFS does not have super-exponential concentration of cylinders. This is because, under this hypothesis, equality $\operatorname{dim}_{A}^{\theta} E=\operatorname{dim}_{B} E$ holds for all $0<\theta<1$ by [12, Corollary 4.2], and also $\operatorname{dim}_{q A} F=\lim _{\theta \rightarrow 1} \operatorname{dim}_{A}^{\theta} F$ for any $F \subset \mathbf{R}$ by [7, Corollary 2.2].

The super-exponential concentration of cylinders property was introduced by Hochman in his celebrated paper [18] to give a substantial improvement on the folklore conjecture that, in absence of exact overlaps, the Hausdorff (and box) dimension of a self-similar set coincides with the minimum between its similarity dimension and 1. He proved that if the conjecture does not hold, then there is super-exponential concentration of cylinders. This property is verified (trivially) in the case that the IFS produces exact overlaps. However, super-exponential concentration does not imply exact overlaps, as was recently shown independently in [1] and [2].

Although difficult to check in general, there are overlapping examples that do not have super-exponential concentration. An example is given by the IFS $\{x / 4,(x+$ $3) / 4,(x+t) / 4\}$. This IFS does not have super-exponential concentration for any $t \in \mathbf{R} \backslash \mathbf{Q}$ (by the same proof of [18, Theorem 1.6]), and $t$ can be chosen so that the WSP does not hold (see the example in [8, Section 4.1]). In particular, there are examples of self-similar sets with box dimension smaller than 1 , that do not verify the WSP but also do not have super-exponential concentration of cylinders. This implies that for the quasi-Assouad dimension there is no such dichotomy as that mentioned above for the Assouad dimension case. 


\section{Further properties}

3.1. Dimensions of sequences with decreasing gaps. In [17] it was shown that sequences in $\mathbf{R}$ with decreasing gaps have Assouad dimension 0 or 1 . It is easy to see that the same statement is true for the quasi-Assouad dimension.

Proposition 9. (i) If $\overline{\operatorname{dim}}_{B} E=0$, then $\operatorname{dim}_{q A} E=0$.

(ii) If $E=\left\{a_{j}\right\}_{j} \subseteq[0,1]$, where $\left\{a_{j}-a_{j+1}\right\}_{j}$ is a decreasing sequence, then $\operatorname{dim}_{q A} E=1$ if $\overline{\operatorname{dim}}_{B} E>0$ and otherwise $\operatorname{dim}_{q A} E=0$.

Proof. (i) Fix $\varepsilon, \delta>0$. The assumption that $\overline{\operatorname{dim}}_{B} E=0$ ensures that for all sufficiently small $r, N_{r}(E) \leq r^{-\delta \varepsilon}$. Thus for any $R \geq r^{1-\delta}$ and any $x \in E$, we have

$$
N_{r}(B(x, R) \cap E) \leq N_{r}(E) \leq r^{-\delta \varepsilon} \leq\left(\frac{R}{r}\right)^{\varepsilon}
$$

from whence the conclusion is immediate.

(ii) In [12, Thm. 6.2] it is shown that if $E$ is a sequence with decreasing gaps, then for all $\theta \in(0,1), \operatorname{dim}_{A}^{\theta} E=\min \left(\frac{\overline{\operatorname{dim}}_{B} E}{1-\theta}, 1\right)$. Thus if $\overline{\operatorname{dim}}_{B} E>0$, then $\operatorname{dim}_{q A} E \geq$ $\sup _{\theta<1} \operatorname{dim}_{A}^{\theta} E=1$.

Remark 5. Although it is also true that $\operatorname{dim}_{A} E=0$ or 1 for sequences with decreasing gaps, the criterion is different. Indeed, as noted in [13, Ex. 6.3], $E=$ $\left\{e^{-\sqrt{n}}\right\}_{n}$ is a set with decreasing gaps having $\overline{\operatorname{dim}}_{B} E=0=\operatorname{dim}_{q A} E, \operatorname{but}_{\operatorname{dim}_{A}} E=1$.

3.2. Quasi-lower Assouad dimension and Hausdorff dimension. The following proposition establishes the relation between these dimensions.

Proposition 10. If $E$ is a closed subset of $\mathbf{R}^{d}$, then $\operatorname{dim}_{q L} E \leq \operatorname{dim}_{H} E$.

Proof. Our proof is based on the method of proof of [20, Theorem 6]. If $\operatorname{dim}_{q L} E=$ 0 there is nothing to prove, so assume $\alpha<\operatorname{dim}_{q L} E$ for some $\alpha>0$ and pick any small $\delta>0$ such that $\alpha<\underline{h_{E}}(\delta)$. We will show $\operatorname{dim}_{H} E \geq \alpha /(1+\delta)$. This will prove the proposition.

Recall that the $r$-packing number of a subset $F \subset \mathbf{R}^{d}, P_{r}(F)$, is the maximum number of disjoint balls of radius $r$ centred in $F$. It is easily seen that there is a constant $c>0$ such that for any bounded $F \subseteq \mathbf{R}^{d}$ and $r>0$, we have $P_{2 r}(F) \geq$ $c N_{r}(F)$. Therefore, since $\alpha<\underline{h_{E}}(\delta)$, there is $\rho_{\delta}>0$ such that for any $x \in E$ and any $r \leq R^{1+\delta} \leq R \leq \rho_{\delta}$

$$
P_{2 r}(B(x, R) \cap E) \geq(R / r)^{\alpha} .
$$

In particular, $P_{2 R^{1+\delta}}(B(x, R) \cap E) \geq R^{-\delta \alpha}$.

Fix $x \in E$ and $R_{1} \leq \rho_{\delta}$. There are $x_{1}, \ldots, x_{R_{1}^{-\delta \alpha}}$ points in $E \cap B\left(x, R_{1}\right)$ such that the balls $B\left(x_{j}, 2 R_{1}^{1+\delta}\right)$ are disjoint for $j=1, \ldots, R_{1}^{\delta \alpha}$. Now let $R_{2}=R_{1}^{1+\delta}$ and notice that

$$
B\left(x_{j}, 2 R_{2}\right) \subseteq B\left(x, R_{1}+2 R_{1}^{1+\delta}\right) \subseteq B\left(x, 2 R_{1}\right)
$$

(as we can take $2 R_{1}^{\delta}<1$ ). We let $C_{0}=B\left(x, 2 R_{1}\right), C_{1}=\bigcup_{j=1}^{R_{1}^{-\delta \alpha}} B\left(x_{j}, 2 R_{2}\right)$ and refer to the balls $B\left(x_{j}, 2 R_{2}\right)$ as the Cantor balls of level 1 .

Repeating this procedure, we see that for each $j$,

$$
P_{2 R_{2}^{1+\delta}}\left(B\left(x_{j}, R_{2}\right) \cap E\right) \geq R_{2}^{-\delta \alpha}
$$


so there are $x_{j, 1}, \ldots, x_{j, R_{2}^{-\delta \alpha}} \in B\left(x_{j}, R_{2}\right) \cap E$, such that $\left\{B\left(x_{j, k}, 2 R_{2}^{1+\delta}\right)\right\}_{k=1}^{R_{2}^{-\delta \alpha}}$ are pairwise disjoint. Put $R_{3}=2 R_{2}^{1+\delta}$. Furthermore,

$$
B\left(x_{j, k}, 2 R_{3}\right) \subseteq B\left(x_{j}, R_{2}+2 R_{3}\right) \subseteq B\left(x_{j}, 2 R_{2}\right) \subseteq C_{1},
$$

so all these balls are disjoint. Let

$$
C_{2}=\bigcup_{k=1}^{R_{2}^{-\delta \alpha}} \bigcup_{j=1}^{R_{1}^{-\delta \alpha}} B\left(x_{j, k}, 2 R_{3}\right)
$$

and call these the Cantor balls of level 2 .

Inductively, given disjoint balls $B\left(x_{j_{1}, \ldots, j_{k-1}}, 2 R_{k}\right)$, then, for $l=1, \ldots, R_{k}^{-\delta \alpha}$, we find points

$$
x_{j_{1}, \ldots, j_{k-1}, l} \in B\left(x_{j_{1}, \ldots, j_{k-1}}, R_{k}\right) \cap E
$$

such that $\left\{B\left(x_{j_{1}, \ldots, j_{k-1}, l}, 2 R_{k}^{1+\delta}\right)\right\}_{l}$ are disjoint. Put $R_{k+1}=R_{k}^{1+\delta}$ and let

$$
C_{k}=\bigcup_{\substack{j_{i} \in\left\{1, \ldots, R_{i}^{-\delta \alpha}\right\} \\ i=1, \ldots, k}} B\left(x_{j_{1}, \ldots, j_{k}}, 2 R_{k+1}\right) \subseteq C_{k-1},
$$

$C_{k}$ being a union of balls of level $k$. where

We have $R_{k+1}=R_{k}^{1+\delta}=R_{1}^{(1+\delta)^{k}}$. Also, notice $C_{k}$ is disjoint union of $M$ balls,

$$
M=\prod_{j=1}^{k} R_{j}^{-\delta \alpha}=\prod_{j=1}^{k} R_{1}^{-\delta \alpha(1+\delta)^{j-1}}=R_{1}^{-\alpha\left((1+\delta)^{k}-1\right)} .
$$

Let $C=\bigcap_{k=1}^{\infty} C_{k}$. As each element of $C$ is a limit point of the centre of the Cantor balls and $E$ is closed, then $C \subseteq E$. We will use the mass distribution principle to check $\operatorname{dim}_{H} C \geq \alpha /(1+\delta)$; see [4, Proposition 2.1].

Let $\mu$ be the probability measure that assigns equal mass on the Cantor balls of each level, i.e., each ball in $C_{k}$ gets measure $M^{-1}=R_{1}^{\alpha\left((1+\delta)^{k}-1\right)}$. We want to show that there is some constant $A=A(\alpha, E)$ such that $\mu(U) \leq A(\operatorname{diam}(U))^{\frac{\alpha}{1+\delta}}$ for all Borel sets $U$.

Without lost of generality we assume $U=B(y, r)$, where $2 R_{k+1}<r \leq 2 R_{k}$, $y \in E$. Any ball of radius $2 R_{k}$ that intersects $U$ will have its centre in $B\left(y, 4 R_{k}\right)$. Let $A_{d}$ be the constant depending on the space $\mathbf{R}^{d}$ such that $P_{2 R_{k}}\left(B\left(y, 4 R_{k}\right)\right) \leq A_{d}$ for all $y \in E$ and all $k$. As Cantor balls at level $k-1$ are disjoint, of radius $2 R_{k}$ and centred in $E$, at most $A_{d}$ of such balls can intersect $U$. Thus $U$ intersects at most $A_{d} R_{k}^{-\delta \alpha}$ level $k$ Cantor balls, so

$$
\mu(U) \leq A_{d} R_{k}^{-\delta \alpha} \cdot R_{1}^{\alpha\left((1+\delta)^{k}-1\right)}=\frac{A_{d}}{R_{1}^{\alpha}} R_{1}^{\alpha(1+\delta)^{k-1}} \leq A(\operatorname{diam}(U))^{\frac{\alpha}{1+\delta}} .
$$

Hence the mass distribution principle implies $\alpha /(1+\delta) \leq \operatorname{dim}_{H} C \leq \operatorname{dim}_{H} E$, completing the proof.

3.3. Different values for different dimensions. Given any six numbers in $[0,1]$, appropriately ordered, there is a compact set $E \subseteq[0,1]$ which have those numbers as the Assouad-type and box dimensions. For simplicity in the exposition, we assume that the upper and lower box dimensions do not coincide and construct Cantor sets to illustrate this. The case in which the box dimension exists is more involved and will be not treated here. 
We make use of the following formulas for the box and quasi-Assouad dimensions of a Cantor set $E$ with ratios of dissection $r_{k}$ at step $k$; see [17] and [21].

$$
\overline{\operatorname{dim}}_{B} E=\limsup _{n} \frac{n \log 2}{\left|\log r_{1} \cdots r_{n}\right|}, \quad \operatorname{dim}_{A} E=\limsup _{n} \sup _{k} \frac{n \log 2}{\left|\log r_{k+1} \cdots r_{k+n}\right|} .
$$

If inf $r_{k}>0$, then

$$
\operatorname{dim}_{q A} E=\lim _{\delta \rightarrow 0} \limsup _{n} \sup _{k \in S_{n, \delta}} \frac{n \log 2}{\left|\log r_{k+1} \cdots r_{k+n}\right|},
$$

where $S_{n, \delta}=\left\{k: r_{k+1} \cdots r_{k+n} \leq\left(r_{1} \cdots r_{k}\right)^{\delta}\right\}$. For the lower box and (quasi)-lower Assouad dimensions replace sup and lim sup by inf and lim inf respectively.

Example 11. Assume $1 \leq a \leq \alpha \leq u<v \leq \beta \leq b<\infty$ are given. There is a Cantor set $E \subseteq[0,1]$ with $\operatorname{dim}_{A} E=1 / a, \operatorname{dim}_{q A} E=1 / \alpha, \overline{\operatorname{dim}}_{B} E=1 / u$, $\underline{\operatorname{dim}}_{B} E=1 / v, \operatorname{dim}_{q L} E=1 / \beta$ and $\operatorname{dim}_{L} E=1 / b$.

The example we will construct is a generalization of [21, Ex. 1.18] and so we will only sketch the ideas. To begin, we choose a sequence of integers $s_{j}$ tending to infinity very rapidly. For convenience, put $t_{2 j}=s_{2 j}\left(\frac{v-\alpha}{u-\alpha}\right)$ and $t_{2 j+1}=s_{2 j+1}\left(\frac{\beta-u}{\beta-v}\right)$. (If $u=\alpha$ put $t_{2 j}=s_{2 j}$ and similarly if $v=\beta$.) We will define the ratios of dissection at the various steps as follows:

$$
\begin{array}{cc}
\text { Ratio } & \text { At steps } \\
2^{-v} & t_{2 j-1}+j, \ldots, s_{2 j} \\
2^{-\alpha} & s_{2 j}+1, \ldots, t_{2 j} \\
2^{-a} & t_{2 j}+1, \ldots, t_{2 j}+j \\
2^{-u} & t_{2 j}+j+1, \ldots, s_{2 j+1} \\
2^{-\beta} & s_{2 j+1}+1, \ldots, t_{2 j+1} \\
2^{-b} & t_{2 j+1}+1, \ldots, t_{2 j+1}+j
\end{array}
$$

Provided $s_{j}$ tends to infinity sufficiently quickly, the ratios at steps $\left\{t_{2 j}+1, \ldots, t_{2 j}+j\right\}$ and $\left\{t_{2 j+1}+1, \ldots, t_{2 j+1}+j\right\}$ will not influence the long run averages that determine the box and quasi-(lower) Assouad dimensions. But these ratios will determine the (lower) Assouad dimensions. The construction ensures that the quasi-Assouad dimension is determined by choosing $R$ to be the length of the Cantor intervals at step $s_{2 j}$ and $r$ to be the length of Cantor intervals at step $t_{2 j}$, while the quasi-lower dimensions arise with $R$ the length at step $s_{2 j+1}$ and $r$ the length at step $t_{2 j+1}$. The choice of $t_{2 j}$ and $t_{2 j+1}$ are made to ensure that the geometric means of the ratios stay within the range $\left[2^{-v}, 2^{-u}\right.$ ] (in the limit) so that the box dimensions are determined along the subsequences of lengths of Cantor intervals at steps $s_{j}$. The details are left to the reader.

3.4. Dimensions of orthogonal projections. For our last example, we will show that, as in the case of Assouad dimension (see [6] and also [11]), the quasiAssouad dimension may increase under orthogonal projections. As before, we will let $\pi_{x}$ (resp., $\pi_{y}$ ) denote the projection onto the $x$ (resp. $y$ ) axis.

Proposition 12. There is a subset $E \subseteq \mathbf{R}^{2}$ such that

$$
\operatorname{dim}_{q A} \pi_{x}(E)=1>1 / 2=\operatorname{dim}_{q A} E .
$$

Proof. We will construct an example to show this. For each $j$ and $i=1, \ldots, 2^{j}$, let $x_{i j}=2^{-j}+(i-1) 2^{-2 j}$. The points $x_{i j}$ belong to $\left[2^{-j}, 2^{-(j-1)}\right)$ and are spaced $2^{-2 j}$ apart. Let $y_{i j}, i=1, \ldots, 2^{j}$, be the endpoints, ordered from left to right, of the 
gaps created at step $j$ in the standard construction of the Cantor set with ratio of dissection $1 / 4$; these gaps have length $2^{-2 j+1}$. Put $E_{j}=\left\{\left(x_{i j}, y_{i j}\right): i=1, \ldots, 2^{j}\right\}$ and $E=\cup_{j} E_{j}$. See Figure 1 below. Of course, $\pi_{x}(E)=\bigcup_{j}\left\{x_{i j}: i=1, \ldots, 2^{j}\right\}$. By checking $N_{r}\left(B\left(x_{0}, R\right) \cap \pi_{x}(E)\right)$ for $x_{0}=2^{-j}, R=2^{-j}$ and $r=2^{-2 j}$, it is easy to see that $\operatorname{dim}_{q A} \pi_{x}(E)=1$.

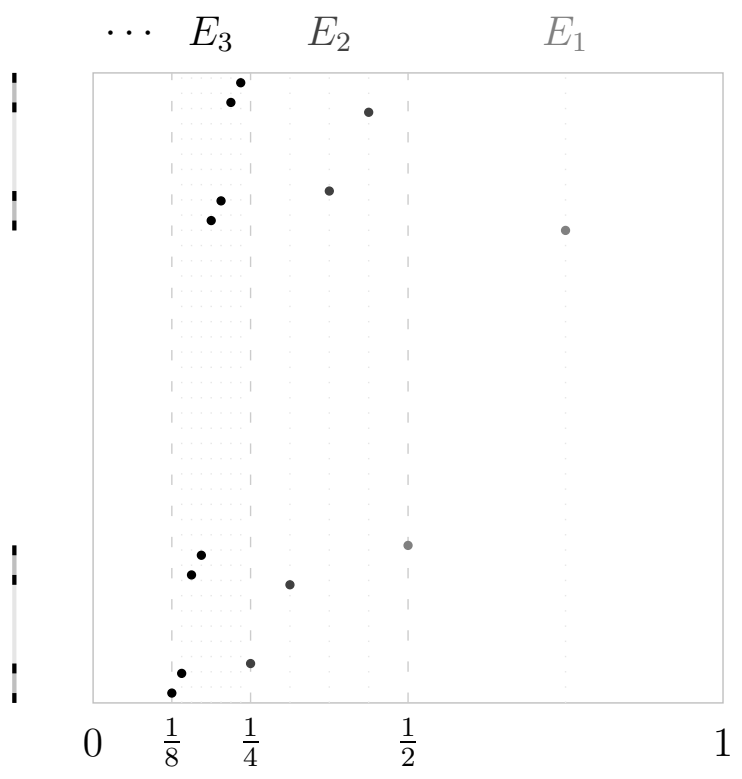

Figure 1. The sets $E_{1}, E_{2}$ and $E_{3}$ in the construction of $E$.

To determine the quasi-Assouad dimension of $E$, it is convenient to take as the definition of a 'ball', $B\left(x_{0}, R\right)$ in $\mathbf{R}^{2}$, the square with centre $x_{0}$ and sides of length $R$. Fix such a ball with $x_{0}=\left(x_{i j}, y_{i j}\right) \in E$ and assume $2^{-2(s+1)}<R \leq 2^{-2 s}$ for some $s \in \mathbf{N}$. The size of $R$ ensures that the interval $\pi_{y}\left(B\left(x_{0}, R\right)\right)$ can intersect only one Cantor interval of step $s$. Choose any $r<R$, say $2^{-2 t}<r \leq 2^{-2(t-1)}$.

First, note that

$$
\left(B\left(x_{0}, R\right) \cap E\right) \cap\left(\left[0,2^{-2 t}\right) \times[0,1]\right):=\Omega
$$

is contained in $\left[0,2^{-2 t}\right) \times\left(\right.$ union of Cantor intervals of step $t$ contained in $\pi_{y}\left(B\left(x_{0}, R\right)\right)$. There are at most $2^{t-s}$ such Cantor intervals, each of length $2^{-2 t}$. Hence $N_{r}(\Omega) \leq$ $2^{t-s}$.

Next, for each $m \in\{t+1, \ldots, 2 t\}$ consider the elements of

$$
\left(B\left(x_{0}, R\right) \cap E\right) \cap\left(\left[2^{-m}, 2^{-(m-1)}\right) \times[0,1]\right):=\Omega_{m} .
$$

The $y$-coordinates of these points are the endpoints of the gaps at step $m$ lying within the one Cantor interval of step $s$ that $\pi_{y}\left(B\left(x_{0}, R\right)\right)$ intersects. There are $2^{m-t}$ of these contained within each Cantor sub-interval of step $t$. As the distance between consecutive $x$-coordinates is $2^{-2 m}$, the total horizontal distance between the points whose $y$-coordinates lie in a (fixed) Cantor interval of step $t$ is $2^{-2 m}\left(2^{m-t}\right)=$ $2^{-(m+t)} \leq 2^{-2 t}$, while the total vertical distance is the width of the Cantor subinterval, $2^{-2 t}$. Consequently, such points lie within a square of side length $2^{-2 t}$ and hence we can cover $\Omega_{m}$ with $2^{t-s}$ squares of side length $2^{-2 t}$ for each such $m$.

Finally, observe that the cardinality of the remainder of $B\left(x_{0}, R\right) \cap E$, which is contained in $\left[2^{-t}, 1\right] \times[0,1]$, is dominated by 2 times the number of gaps of step $\leq t$ within a Cantor interval of step $s$, and this is bounded above by $2^{t-s+1}$. Combining 
together all these observations, we see that

$$
N_{r}\left(B\left(x_{0}, R\right) \cap E\right) \leq(t+1) 2^{t-s}+2^{t-s+1} .
$$

It follows that for each $\delta>0, \overline{h_{E}}(\delta) \leq 1 / 2$ and thus $\operatorname{dim}_{q A} E \leq 1 / 2$. It is not difficult to see that these estimates are essentially sharp and thus we actually have $\operatorname{dim}_{q A} E=1 / 2$.

Remark 6. The classical Marstrand projection theorem, and its more recent variants, (cf. [5]) states that the orthogonal projections of planar sets have the same dimension at almost every angle, where here dimension can be Hausdorff, upper/lower box or packing. This is not the case for the Assouad dimension. Indeed, it is shown in [11] that for any $s$ with $\log _{5} 3<s<1$, there exists a self-similar set $F \subset \mathbf{R}^{2}$ and two non-empty intervals $I, J$ such that $\operatorname{dim}_{A} \pi_{\theta} F=s$ for all $\theta \in I$, while $\operatorname{dim}_{A} \pi_{\theta} F=1$ for almost all $\theta \in J$. Here $\pi_{\theta}$ denotes the projection onto the line passing through the origin with angle $\theta$. Indeed, the situation can be more dramatic. It is shown in [10] that the Assouad dimension of orthogonal projections can take on a countable number of distinct values on a set of projections with positive measure.

It is unknown what the situation is for the quasi-Assouad dimension. The set $F$ from [11] does not seem to be helpful for resolving this problem. Since it is a Sierpinski triangle with contraction factor $c$, for some $c \in(1 / 5,1 / 3)$, its orthogonal projections are self-similar sets $F_{t}$ attractors of iterated functions systems of the form $\mathcal{I}_{c, t}=\{c x, c x+1-c, c x+t\}$, up to rescaling. Then, if $c$ is algebraic, for almost every $t$, these projections does not have super exponential contraction of cylinders (see Theorem 1.6 in [18] and the comment after its proof). Hence by Remark 4, for almost every $t$ we have $\operatorname{dim}_{q A} F_{t}=\operatorname{dim}_{B} F_{t}$, and thus the Marstrand projection theorem implies that the quasi-Assouad dimensions of the projections of $F$ are constant almost everywhere. On the other hand, if $c$ is not algebraic, it is unknown in general if there are super exponential contraction of cylinders in the projections, so their quasi-Assouad dimension is unknown. Note that there are uncountably many pairs $(c, t)$ such that for $\mathcal{I}_{c, t}$ there are no exact overlaps but exponential concentration of cylinders; see [2, Theorem 2.2].

Acknowledgements. The work of I. García was partially supported by a grant from the Simons Foundation. The work of K. Hare was supported by NSERC 2016 03719.

\section{References}

[1] BAKer, S.: Iterated function systems with super-exponentially close cylinders. - Preprint, arXiv:1909.04343, 2019.

[2] BÁRÁNy, B., and A. KÄENMÄKI: Super-exponential condensation without exact overlaps. Preprint, arXiv:1910.04623, 2019.

[3] Chen, C., M. Wu, and W. Wu: Accessible values of Assouad and the lower dimensions of subsets. - Preprint, arXiv:1602.02180, 2016.

[4] Falconer, K.: Techniques in fractal geometry. - John Wiley \& Sons Ltd., Chichester, 1997.

[5] Falconer, K., J. Fraser, and X. Jin: Sixty years of fractal projections. - In: Fractal geometry and stochastics V, Progr. Prob. 70, Birkhauser/Springer, Cham, 2015, 3-25.

[6] Fraser, J.: Assouad type dimensions and homogeneity of fractals. - Trans. Amer. Math. Soc. $336,2014,6687-6783$. 
[7] Fraser, J., K. G. Hare, K. E. Hare, S. Troscheit, and H. Yu: The Assouad spectrum and the quasi-Assouad dimension: a tale of two spectra. - Ann. Acad. Sci. Fenn. Math. 44, 2019, 379-387.

[8] Fraser, J., A. Henderson, E. Olson, and J. Robinson: On the Assouad dimension of self-similar sets with overlaps. - Adv. Math. 273, 2015, 188-214.

[9] Fraser, J., D. Howroyd, A. Käenmäki, and H. Yu: On the Hausdorff dimension of microsets. - Proc. Amer. Math. Soc. 147:11, 2019, 4921-4936.

[10] Fraser, J., and A. KÄEnmäKI: Attainable values for the Assouad dimension of projections. - Proc. Amer. Math. Soc. 148, 2020, 3393-3405.

[11] Fraser, J., and T. Orponen: The Assouad dimensions of projections of planar sets. - Proc. London Math. Soc. 114:2, 2017, 374-398.

[12] Fraser, J. M., and H. Yu: Assouad type spectra for some fractal families. - Indiana Univ. Math. J. 67:5, 2018, 2005-2043.

[13] Fraser, J., and H. Yu: New dimension spectra: finer information on scaling and homogeneity. - Adv. Math. 329, 2018, 273-328.

[14] Fraser, J., and H. Yu: Arithmetic patches, weak tangents, and dimension. - Bull. Lond. Math. Soc. 50:1, 2018, 85-95.

[15] Furstenberg, H.: Ergodic fractal measures and dimension conservation. - Ergodic Theory Dynam. Systems 28:02, 2008, 405-422.

[16] Furstenberg. H.: Ergodic theory and fractal geometry. - CBSM Reg. Conf. Ser. Math. 120, Amer. Math. Soc., 2014.

[17] García, I., K. E. Hare, and F. Mendivil: Assouad dimensions of complementary sets. Proc. Roy. Soc. Edinburgh Sect. A 148, 2018, 517-540.

[18] Hochman, M.: On self-similar sets with overlaps and inverse theorems for entropy. - Ann. of Math. (2) 180, 2014, 773-822.

[19] Käenmäki, A., T. Ojala, and E. Rossi: Rigidity of quasisymmetric mappings on self-affine carpets. - Int. Math. Res. Not. IMRN 2018:12, 2017, 3769-3799.

[20] Larman, D. G.: On Hausdorff measure in finite-dimensional compact metric spaces. - Proc. Amer. Math. Soc. 17, 1967, 193-206.

[21] LÜ, F., and L. XI: Quasi-Assouad dimension of fractals. - J. Fractal Geom. 3:2, 2016, $187-215$.

[22] MackAy, J.: Assouad dimension of self-affine carpets. - Conform. Geom. Dyn. 15:12, 2011, 177-187.

[23] MACKAY, J., and J. Tyson: Conformal dimension: theory and applications. - Univ. Lecture Ser. 54, 2010.

[24] Zerner, M.: Weak separation properties for self-similar sets. - Proc. Amer. Math. Soc. 124:11, 1996, 3529-3539.

Received 10 July $2019 \bullet$ Accepted 29 April 2020 